\title{
Influence of the relative age effect on children's scores obtained from the Canadian assessment of physical literacy
}

\author{
Caroline Dutil ${ }^{1,2}$, Mark S. Tremblay ${ }^{2}$, Patricia E. Longmuir ${ }^{1,2}$, Joel D. Barnes², Kevin Belanger ${ }^{2}$ \\ and Jean-Philippe Chaput ${ }^{1,2^{*}}$
}

\begin{abstract}
Background: Age grouping by the imposition of a cut-off date, common in sports and education, promotes a relative age difference that is associated with developmental advantages for children who are born on the "early side" of the cut-off date and disadvantages to those born later in the same year, which is known as the relative age effect (RAE) bias. Acquiring an adequate level of physical literacy is important for children to remain active for life. The Canadian Assessment of Physical Literacy (CAPL) is an assessment protocol that encompasses measures in the domains of children's Daily Behaviours, Physical Competence, Motivation and Confidence, and Knowledge and Understanding. The purpose of this study was to ascertain whether the CAPL scores were susceptible to the RAE, which could affect our interpretation of the CAPL findings.
\end{abstract}

Methods: This cross-sectional study examined if scores obtained in the CAPL (i.e., the four domains individually and the total CAPL score) were susceptible to the RAE in children aged 8 to 12 years and, if so, which physical competence assessments (movement skills, cardiorespiratory, strength, muscular endurance, flexibility, and body composition measurements) were more susceptible. Participants ( $n=8233,49.8 \%$ boys) from the Royal Bank of Canada-CAPL Learn to Play project from 11 sites in seven Canadian provinces were tested using the CAPL protocol.

Results: Among boys and girls, the RAE was significantly associated with two and three of the four domain scores, respectively, after controlling for covariates. However, effect sizes were negligible for the comparisons between quarters of the year and physical literacy domains and overall scores. For the main effect of the relative age, boys and girls born in the first three months of the year were taller $\left(F(3,4074)=57.0, p<0.001, f^{2}=0.04\right.$ and $F(3,4107)=$ $58.4, p<0.001, f^{2}=0.04$, respectively) and demonstrated greater muscular strength $\left(F(3,4037)=29.2, p<0.001, f^{2}=\right.$ 0.02 and $F(3,4077)=25.1, p<0.001, f^{2}=0.02$, respectively) compared with those born later in the same year.

Conclusions: Collectively, our results suggest that the RAE bias is mainly negligible with regard to the domain scores and overall CAPL scores in this large sample of children.

Keywords: Movement skills, Health behaviours, Motivation, Physical activity, Month of birth bias, Pediatric

\footnotetext{
* Correspondence: jpchaput@cheo.on.ca

${ }^{1}$ School of Human Kinetics, Faculty of Health Sciences, University of Ottawa,

Ottawa, ON K1N 6N5, Canada

${ }^{2}$ Healthy Active Living and Obesity Research Group, Children's Hospital of

Eastern Ontario Research Institute, 401 Smyth Road, Ottawa, ON K1H 8L1,

Canada
}

(c) The Author(s). 2018 Open Access This article is distributed under the terms of the Creative Commons Attribution 4.0 International License (http://creativecommons.org/licenses/by/4.0/), which permits unrestricted use, distribution, and reproduction in any medium, provided you give appropriate credit to the original author(s) and the source, provide a link to the Creative Commons license, and indicate if changes were made. The Creative Commons Public Domain Dedication waiver (http://creativecommons.org/publicdomain/zero/1.0/) applies to the data made available in this article, unless otherwise stated. 


\section{Background}

Physical literacy is a concept that has gained momentum globally; it is defined by the International Physical Literacy Association as "the motivation, confidence, physical competence, knowledge and understanding to value and take responsibility for engagement in physical activity for life" [1]. The goal of physical literacy (i.e., lifelong engagement in physical activity) is an attractive idea, especially when considering how critical physical activity is for children's health and well-being [2]. Many countries have adopted and included the physical literacy construct in their education system, and sports governing bodies have also followed suit in their athlete development programs [3-5].

In response to the need to assess physical literacy in children, the Canadian Assessment of Physical Literacy (CAPL) was recently developed and validated $[6,7]$. The CAPL is a comprehensive measurement tool that encompasses many aspects within the four relevant domains of physical literacy (Physical Competence, Daily Behaviour, Motivation and Confidence, and Knowledge and Understanding) and provides an overall physical literacy score for 8- to 12-year-old children $[6,7]$. The CAPL's four domains are aligned with the current physical literacy definition $[1,6,7]$.

In sports and education systems, children are commonly grouped by age as an administrative strategy in order to provide age-adjusted competition and learning opportunities. However, age grouping by the imposition of a cut-off date promotes a relative age difference that often leads to developmental advantages for children who are born on the "early side" of the cut-off date. These differences are known as the relative age effect (RAE) bias [8-11]. Indeed, those who are disadvantaged by the RAE are underrepresented at the elite and professional level in many sports [10], are at greater risk of having inferior grades in school [12-15], demonstrate lower levels of self-efficacy [16], display poorer mental health coping mechanisms [17], are at increased risk of needing special education support [18], are at greater risk of dropping out from sports $[19,20]$, and are at greater risk of being diagnosed with attention deficit hyperactivity disorder [21].

Studies on the RAE have also expanded to the testing of fitness, fundamental movement and developmental skills, where children are often compared using normative data that group children into rounded-down age bands (e.g., where a 9.9-year-old may be compared to a 9.1-year-old in an age band for all 9-year-old children). It is no surprise that an association between fitness, developmental and fundamental movement skill assessments and the RAE have been found, since this form of age grouping is analogous to the cohort grouping seen in sports and education systems [11, 22-24]. However, no study to date has examined the RAE in the context of physical literacy, a construct that simultaneously takes into account affective, cognitive, behavioural, and physical measurements. The present study was conducted to address this knowledge gap, and to assess the magnitude of the RAE using a comprehensive physical literacy assessment protocol.

The CAPL is scored by separating children into rounded-down age bands that span a minimum of a full year. Furthermore, if education systems and sports governing bodies are delivering physical literacy programs, school entry and sports cut-off dates become important factors in children's acquisition of physical literacy skills, especially considering that cut-off dates are similar across many sports and education systems in many developed countries [10].

Thus, the present study aimed to examine the patterns of association between month of birth (relative age) and physical literacy assessment scores (i.e., individual domain scores and the total CAPL scores) in children aged 8 to 12 years. We also examined the magnitude of the $\mathrm{RAE}$ in the different components within the physical competence domain (i.e., movement skills, cardiorespiratory fitness, strength, muscular endurance, flexibility, and body composition measurements). We hypothesized that children born earlier in the year would score higher on physical literacy components than those born later in the same calendar year.

\section{Methods \\ Participants}

The Royal Bank of Canada (RBC) CAPL Learn to Play project was a cross-sectional study comprising a large number of Canadian children between 8 and 12 years of age. The aim was to recruit 12,500 children total from 11 Canadian sites: Victoria, British Columbia; Lethbridge, Alberta; Calgary, Alberta; Winnipeg, Manitoba; North Bay, Ontario; Windsor, Ontario; Ottawa, Ontario; Trois-Rivières, Québec; Halifax, Nova Scotia; Antigonish, Nova Scotia; and Charlottetown, Prince Edward Island. Although this was a convenience sample, testing sites were instructed to recruit children in a variety of settings (e.g., elementary schools, after-school programs and community centres) from urban, suburban and rural areas in and around their region, while also trying to ensure a balanced representation of high-, medium-, and low-income communities. Participants were tested by trained appraisers between February 2014 and February 2017. A parent (or legal guardian) provided written consent for participation in the study and completed a screening form indicating that their child had no known limitations for physical activity, including maximal effort exercise. Children also gave assent to partake in this assessment, and the Ethics review boards at each participating institution also approved the protocol. 


\section{Study protocol}

Physical literacy was assessed using the CAPL protocol. Longmuir and colleagues [7] have published a detailed explanation of the CAPL protocol, including its validity. The CAPL is also available online (www.capl-eclp.ca), and includes a detailed manual, training videos, and other relevant information that can be accessed or downloaded, in either English or French, for free [25]. The CAPL instrument measures, which are consistent with the current definition of physical literacy by the International Physical Literacy Association, assess each of the four domains of physical literacy (Physical Competence, Daily Behaviour, Knowledge and Understanding, and Motivation and Confidence), and provide an overall composite physical literacy score (i.e., total CAPL score) $[6,7]$.

A Delphi expert panel process was used to inform the CAPL scoring system. The total CAPL score (maximum of 100 points) is a composite sum of the scores obtained in the four domains, where both the Physical Competence and the Daily Behaviour domains are more heavily weighted (32 points each) than the Knowledge and Understanding and the Motivation and Confidence domains (18 points each) (see Additional file 1) [6, 7]. For more details on Canada's physical literacy consensus statement, process, outcomes, and normative data, see Tremblay and colleagues [26, 27]. A short explanation of each domain is provided below.

\section{Physical competence domain}

The aim of the Physical Competence domain is to test children's physical core competencies to partake in physical activities by assessing their physical fitness, movement skills, and body composition. The score for this domain is composed of objective measurements of body composition (body mass index [BMI] $z$-score [28] and waist circumference [WC] [29]), cardiorespiratory fitness (Progressive Aerobic Cardiovascular Endurance Run [PACER] shuttle run) [30], muscular strength (grip strength) [29], muscular endurance (timed plank test) [31], flexibility (sit-and-reach) [29], and movement skills performance (Canadian Agility and Movement Skill Assessment [CAMSA]) (see Additional file 2) [32].

\section{Daily behaviour domain}

The Daily Behaviour domain contains three components: average daily step counts measured via pedometer worn for seven consecutive days, self-reported sedentary time, and self-reported moderate to vigorous physical activity. Pedometer data criteria were established as follows: (i) step counts between 1000 and 30,000 steps daily [33]; (ii) minimum wear time of $10 \mathrm{~h}$ daily [34]; and (iii) at least three days of valid data that meet both aforementioned criteria [35]. The two other components were subjectively assessed via questionnaire, where children were asked to recall how many days in the past week they had engaged in a total of $60 \mathrm{~min}$ or more of moderate to vigorous physical activity, and to self-report their daily screen time habits [25]. For more details on the sedentary behaviour assessment, see Saunders and colleagues [36].

\section{Knowledge and understanding domain}

The Knowledge and Understanding domain was assessed using a questionnaire that was designed to test aspects of healthy behaviour and the knowledge level that is expected based on Canadian physical and health education curricula (for grades 4,5 , and 6) across all provinces/territories [6]. The questions evaluate children's knowledge and understanding of the Canadian Physical Activity and Sedentary Behaviour Guidelines for Children and Youth (http://csepguidelines.ca/children-and-youth-5-17/), related terms, definition of health, recommended safety equipment to partake in certain physical activities and sports, and a basic understanding of how movement skills can be improved [6, 7, 37].

\section{Motivation and confidence domain}

The Motivation and Confidence domain, which was assessed via questionnaire, evaluated children's self-perceived benefits and barriers for physical activity, self-perceived activity and skill levels compared to peers, and their adequacy in and predilection for physical activity $[6,7,38]$. The questions on physical activity barriers and benefits were based on the published scales developed by Garcia and colleagues [39]. Two thirds of this domain score were attributed to children's responses to the adequacy and predilection subscales of the Children's Self-Perceptions of Adequacy in and Predilection for Physical Activity (CSAPPA) questionnaire [40].

\section{Analytic sample}

A flow diagram of the samples used in the present study is shown in Fig. 1. A little over 11,000 online accounts were created for the RBC-CAPL Learn to Play project; of those, any accounts that were outside the CAPL validated age group or that were missing key information were excluded. Any participants with raw scores less than quartile 1 - (1.5 $\mathrm{x}$ the interquartile range) or greater than quartile $3+(1.5 \mathrm{x}$ the interquartile range $)$ were deemed outliers and removed from the dataset. Ageand gender-specific $z$-scores were created, and participants outside $\pm 5 z$-scores were also deemed outliers and removed. Participants were also excluded from the analyses if they (or a parent) reported a medical condition or disability that would likely influence the CAPL assessment scores $(n=36)$ (e.g., broken limb in a cast, Down syndrome, autism, and those who reported acute injury on the day of testing). For the purpose of the present 
12500 RBC-CAPL Learn to Play recruitment goal

11071 online accounts

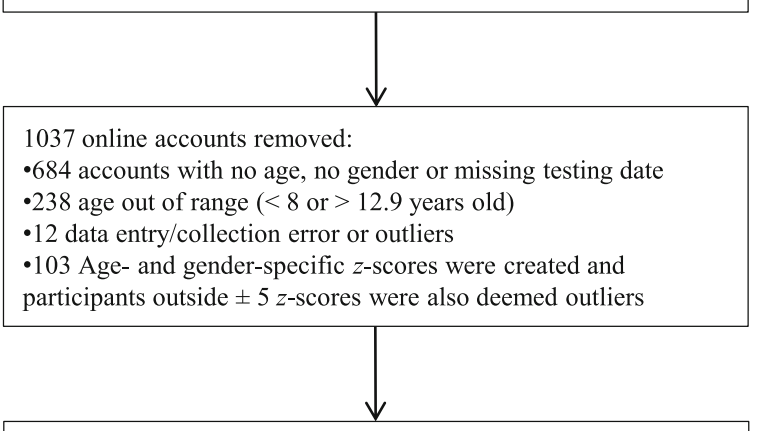

10034 Participants in the RBC-CAPL Learn to Play dataset

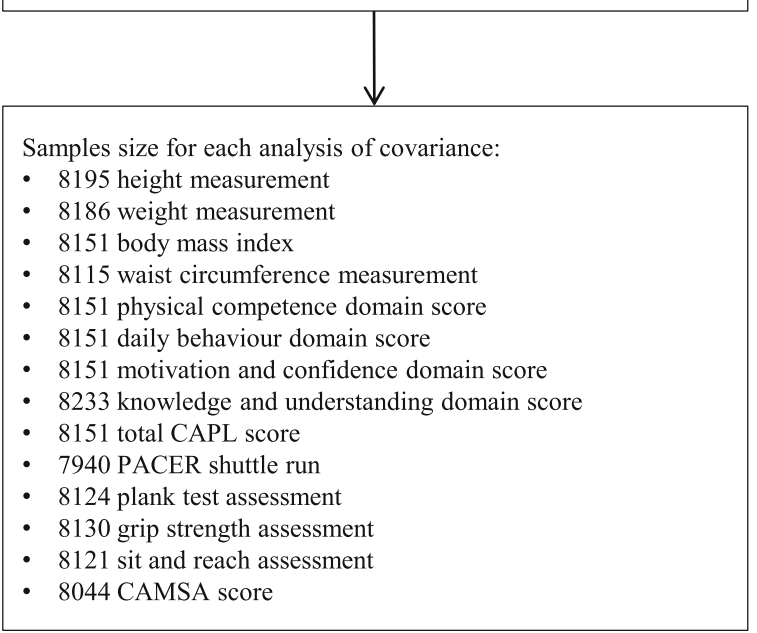

Fig. 1 Sample sizes from recruitment goal to the number of participants in each analysis. Abbreviations: CAMSA Canadian Agility and Movement Skill Assessment, CAPL Canadian Assessment of Physical Literacy, PACER Progressive Aerobic Cardiovascular Endurance Run, RBC Royal Bank of Canada.

paper, those with birth month missing were ineligible and removed $(n=3)$. For all the analyses, those with incomplete scores for the dependent variable of interest were excluded. However, for the domain scores, it is possible to calculate a domain score if one assessment is missing from the domain, so those participants remained in the analytic samples $[6,7,25]$. Descriptive characteristics of children who were excluded did not differ from those who were included in the present analyses.

\section{Statistical analysis}

In the present study, the independent variable was the relative age of the children based on month of birth and school entry cut-off date. The dependent variables were the scores obtained in each individual domain of CAPL as well as the overall CAPL score. Since the Physical Competence domain assesses many different components that may be unequally affected by the RAE, these dependent variables were also assessed individually. Covariates used for this study included age (in whole year), the testing month, the testing sites (geographic location based on the specific testing site), and children's BMI $z$-scores (when relevant to the dependent variable). In order to examine the associations between the RAE bias and the scores obtained on physical literacy, children were stratified into quarters of the year based on their birth month and the annual school entry cut-off date. For all the sites except one, the annual school entry cut-off date is December 31, so for those sites children born in January, February, and March were assigned to quarter 1; children born in April, May, and June were assigned to quarter 2; etc. For the Trois-Rivières site in the province of Québec, the school entry cut-off date is October 1; therefore, we adjusted the quarter grouping for those participants $(n=42)$ accordingly for all analyses of covariance (ANCOVA) (i.e., children born in October, November, and December were assigned to quarter 1; children born in January, February, and March were assigned to quarter 2; etc.). Data were tested for assumptions of normality, linearity, and homogeneity of variance. As a result, no transformations were necessary.

As expected, our sample displayed an interaction effect for gender in the relationship between relative age and performance in CAPL assessments; boys and girls were therefore analyzed separately. However, no interaction was found for age (in whole years) for the same relationship; thus, all ages were analyzed together. We performed ANCOVAs, controlling for the above-mentioned covariates (age, testing month, testing sites and BMI $z$-scores [when relevant to the dependent variable]), with a Bonferroni post-hoc test to identify individual differences in mean scores per quarters. An alpha level of < 0.05 was set to establish statistical significance. Finally, to assess the practical or clinical significance of our findings, we calculated Cohen's $f^{2}$ [41] from the partial $\eta^{2}$ to interpret the effect sizes for our main effect (relative age in quarters). We interpreted the effect sizes according to Cohen's guidelines [41], i.e., $f^{2} \geq 0.02, f^{2} \geq 0.15$ and $f^{2} \geq$ 0.35 representing small, medium and large effect sizes, respectively. All statistical analyses were conducted using IBM SPSS Statistics for Windows, version 24 (IBM Corp., Armonk, NY, USA).

\section{Results}

Tables 1 and 2 show the descriptive characteristics of our participants (49.8\% boys). Birth months divided into quarters were not distributed evenly; almost $30 \%$ of the sample was born in the months of July, August, and September. However, 
Table 1 Descriptive characteristics for children who participated in the RBC-CAPL study

\begin{tabular}{|c|c|c|c|c|c|c|}
\hline & \multicolumn{2}{|c|}{ All } & \multicolumn{2}{|c|}{ Boys } & \multicolumn{2}{|c|}{ Girls } \\
\hline & $n$ & Mean (SD) or \% & $n$ & Mean $(S D)$ or $\%$ & $n$ & Mean (SD) or $\%$ \\
\hline Age (y) & 8233 & $10.6(1.2)$ & 4100 & $10.6(1.2)$ & 4133 & $10.6(1.2)$ \\
\hline Height (cm) & 8195 & $144.1(9.8)$ & 4081 & $144.0(9.6)$ & 4114 & $144.3(10.1)$ \\
\hline Weight (kg) & 8186 & $40.0(11.5)$ & 4076 & $40.0(11.5)$ & 4110 & $40.1(11.4)$ \\
\hline BMI $\left(\mathrm{kg} / \mathrm{m}^{2}\right)$ & 8151 & $19.0(3.8)$ & 4059 & $18.9(3.854)$ & 4092 & $19.0(3.727)$ \\
\hline$W C(\mathrm{~cm})$ & 8115 & $67.3(10.8)$ & 4039 & $67.4(11.0)$ & 4076 & $67.1(10.5)$ \\
\hline Sites (province): & 8233 & 100.0 & 4100 & 100.0 & 4133 & 100.0 \\
\hline Antigonish (NS) & 840 & 10.2 & 410 & 10.0 & 430 & 10.4 \\
\hline Calgary (AB) & 1126 & 13.7 & 564 & 13.8 & 562 & 13.6 \\
\hline Charlottetown (PEI) & 456 & 5.5 & 230 & 5.6 & 226 & 5.5 \\
\hline Halifax (NS) & 648 & 7.9 & 321 & 7.8 & 327 & 7.9 \\
\hline Lethbridge (AB) & 900 & 10.9 & 444 & 10.8 & 456 & 11.0 \\
\hline North Bay (ON) & 966 & 11.7 & 457 & 11.1 & 509 & 12.3 \\
\hline Ottawa (ON) & 619 & 7.5 & 292 & 7.1 & 327 & 7.9 \\
\hline Trois-Rivières (QC) & 42 & 0.5 & 27 & 0.7 & 15 & 0.4 \\
\hline Victoria (BC) & 425 & 5.2 & 225 & 5.5 & 200 & 4.8 \\
\hline Windsor (ON) & 1108 & 13.5 & 578 & 14.1 & 530 & 12.8 \\
\hline Winnipeg (MB) & 1103 & 13.4 & 552 & 13.5 & 551 & 13.3 \\
\hline
\end{tabular}

$A B$ Alberta, $B C$ British Columbia, BMI body mass index, MB Manitoba, NS Nova Scotia, ON Ontario, PEl Prince Edward Island, QC Quebec, RBC Royal Bank of Canada, $S D$ standard deviation, WC waist circumference

our sample was representative of the Canadian birth distribution [42] according to a Chi-square goodness-of-fit test.

The $F$-test values presented in this section represent the main effect of the relative age in quarters or the covariates' contribution to the model, while the ones shown in Tables 3 and 4 are the corrected model $F$-test values. In Table 3, height of boys and girls was significantly associated with the relative age $(F(3,4074)=57.0, p<0.001$ and $F(3,4107)=58.4, p<0.001$, respectively). Cohen's $f^{2}$ effect sizes were considered small for both boys and girls. Moreover, girls' height findings revealed a dose-response association with the RAE (quarter $1>$ quarter $2>$ quarter $3>$ quarter 4). Girls born in quarter 4 (i.e., born between

Table 2 Comparison of our sample's birth distribution with Canadian live birth data between 2002 and 2008

\begin{tabular}{lccc}
\hline Birth months & $\mathrm{n}$ & Sample (\%) & Canadian births (\%) \\
\hline Quarter 1 & 1980 & 24.0 & 23.8 \\
Quarter 2 & 2097 & 25.5 & 25.5 \\
Quarter 3 & 2345 & 28.5 & 26.4 \\
Quarter 4 & 1811 & 22.0 & 24.3 \\
& \multicolumn{4}{c}{$x^{2}=0.39 ; \mathrm{df}=3 ; p=0.94$} \\
\hline
\end{tabular}

A Chi-square goodness-of-fit test was performed to compare our samples' birth month (in quarters) to the average Canadian births by months. The Canadian births are the averages of the percentages of Canadian live births per month between the years 2002 and 2008 [42]. Quarter 1: January-March; Quarter 2: April-June; Quarter 3: July-September; Quarter

4: October-December
October to December) were significantly shorter than girls born in all other quarters. There was no significant difference in height between boys born in the last six months of the year (quarters 3 and 4).

Similarly, weight was associated with the relative age differences $(F(3,4069)=20.6, p<0.001$ and $F(3,4103)=$ $12.7, p<0.0001$, in boys and girls, respectively). The effect sizes for weight were small $\left(f^{2}=0.02\right)$ and negligible $\left(f^{2}=0.01\right)$ in boys and girls, respectively. BMI was significantly associated with the relative age in boys only $(F(3,4052)=5.4, p=0.001$ and $F(3,4085)=0.9, p=0.45$ in boys and girls, respectively), but the effect size was considered negligible. Among boys and girls, the main effect of relative age was associated with the WC measurement $(F(3,4032)=8.7, p<0.001$ and $F(3,4069)=3.1$, $p=0.03$, respectively), but the effect sizes for these associations were also negligible. The covariate age was the largest contributor in the model for height, weight, BMI and WC for both genders (data not shown).

The results for the Physical Competence domain revealed a significant main effect of quarter in both boys and girls $(F(3,4051)=11.8, p<0.0001$ and $F(3,4084)=$ 15.7, $p<0.0001$, respectively), but yielded negligible effect sizes in both boys and girls for the main effect of quarter. However, the covariate BMI $z$-score in the Physical Competence domain score model resulted in large effect sizes in both genders $\left(f^{2}=0.44\right.$ and $f^{2}=0.40$ in boys and girls, respectively). 
Table 3 Relative age differences in scores obtained in the Canadian Assessment of Physical Literacy based on children's month of birth

\begin{tabular}{|c|c|c|c|c|c|c|c|c|c|c|c|c|c|c|c|}
\hline & \multicolumn{3}{|c|}{$\begin{array}{c}\text { Quarter } 1 \\
\text { January to March }\end{array}$} & \multicolumn{3}{|c|}{$\begin{array}{c}\text { Quarter } 2 \\
\text { April to June }\end{array}$} & \multicolumn{3}{|c|}{$\begin{array}{c}\text { Quarter } 3 \\
\text { July to September }\end{array}$} & \multicolumn{3}{|c|}{$\begin{array}{c}\text { Quarter } 4 \\
\text { October to December } \\
\end{array}$} & \multirow[t]{3}{*}{ F-test } & \multirow[b]{3}{*}{$p$} & \multirow[b]{3}{*}{$f^{2}$} \\
\hline & \multirow[t]{2}{*}{ Mean } & \multicolumn{2}{|c|}{$95 \% \mathrm{Cl}$} & \multirow[t]{2}{*}{ Mean } & \multicolumn{2}{|c|}{$95 \% \mathrm{Cl}$} & \multirow[t]{2}{*}{ Mean } & \multicolumn{2}{|c|}{$95 \% \mathrm{Cl}$} & \multirow[t]{2}{*}{ Mean } & \multicolumn{2}{|c|}{$95 \% \mathrm{Cl}$} & & & \\
\hline & & LB & UB & & LB & UB & & LB & UB & & LB & UB & & & \\
\hline \multicolumn{16}{|l|}{ Boys } \\
\hline Height $(\mathrm{cm})^{\mathrm{a}}(n=4081)$ & 146.2 & 145.7 & 146.6 & $144.6 \neq$ & 144.1 & 144.9 & 142.9キ† & 142.5 & 143.3 & $142.3 \neq \dagger$ & 141.9 & 142.8 & $563.86^{*}$ & $<0.001$ & 0.04 \\
\hline Weight $(\mathrm{kg})^{\mathrm{a}}(n=4076)$ & 42.0 & 41.3 & 42.6 & $39.9 \neq$ & 39.3 & 40.5 & $38.9 \neq$ & 38.3 & 39.5 & $38.6 \neq \dagger$ & 37.9 & 39.3 & $175.57^{*}$ & $<0.001$ & 0.02 \\
\hline BMI $\left(\mathrm{kg} / \mathrm{m}^{2}\right)^{\mathrm{a}}(\mathrm{n}=4059)$ & 19.4 & 19.1 & 19.6 & $18.9 \neq$ & 18.6 & 19.1 & $18.8 \neq$ & 18.6 & 19.0 & $18.8 \neq$ & 18.5 & 19.0 & $35.09^{*}$ & 0.001 & 0.004 \\
\hline$W C(\mathrm{~cm})^{\mathrm{a}}(n=4039)$ & 68.9 & 68.2 & 69.5 & $67.4 \neq$ & 66.8 & 68.0 & $67.0 \neq$ & 66.4 & 67.6 & $66.5 \neq$ & 65.8 & 67.2 & $72.28^{*}$ & $<0.001$ & 0.01 \\
\hline $\begin{array}{l}\text { Physical Competence } \\
\text { domain score }{ }^{\mathrm{b}}(n=4059)\end{array}$ & 20.6 & 20.3 & 20.8 & $20.1 \neq$ & 19.9 & 20.3 & $19.8 \neq$ & 19.6 & 20.0 & $19.6 \neq \dagger$ & 19.3 & 19.8 & $277.04^{*}$ & $<0.001$ & 0.01 \\
\hline $\begin{array}{l}\text { Daily Behaviour domain } \\
\text { score }^{\mathrm{b}}(n=4059)\end{array}$ & 18.6 & 18.1 & 19.1 & 18.5 & 18.0 & 18.9 & 18.6 & 18.1 & 19.0 & 19.1 & 18.6 & 19.0 & $12.54^{*}$ & 0.26 & 0.001 \\
\hline $\begin{array}{l}\text { Motivation and Confidence } \\
\text { domain score }(n=4059)\end{array}$ & 12.9 & 12.7 & 13.1 & 12.8 & 12.6 & 12.9 & 12.6 & 12.5 & 12.8 & 12.8 & 12.6 & 12.9 & $5.48^{*}$ & 0.42 & 0.001 \\
\hline $\begin{array}{l}\text { Knowledge and Understanding } \\
\text { domain score }(n=4100)\end{array}$ & 12.2 & 12.0 & 12.4 & 12.0 & 11.9 & 12.2 & $11.7 \neq \dagger$ & 11.5 & 11.8 & $11.6 \neq \dagger$ & 11.5 & 11.8 & $78.49^{*}$ & $<0.001$ & 0.01 \\
\hline $\begin{array}{l}\text { Total CAPL score }{ }^{b} \\
(n=4059)\end{array}$ & 64.2 & 63.4 & 64.9 & 63.3 & 62.6 & 64.1 & $62.7 \neq$ & 61.9 & 63.4 & 63.1 & 62.3 & 63.9 & $60.4^{*}$ & 0.04 & 0.002 \\
\hline \multicolumn{16}{|l|}{ Girls } \\
\hline Height $(\mathrm{cm})^{\mathrm{a}}(n=4114)$ & 146.5 & 146.1 & 147.0 & $144.6 \neq$ & 144.2 & 145.1 & $143.7 \neq \dagger$ & 143.3 & 144.1 & $142.2 \neq \dagger$ & 141.8 & 142.7 & $629.71^{*}$ & $<0.001$ & 0.04 \\
\hline Weight $(\mathrm{kg})^{\mathrm{a}}(n=4110)$ & 41.5 & 40.9 & 42.2 & 40.4 & 39.8 & 41.0 & $39.5 \neq$ & 39.0 & 40.1 & $38.9 \neq \dagger$ & 38.2 & 39.6 & $201.36^{*}$ & $<0.001$ & 0.01 \\
\hline BMI $\left(\mathrm{kg} / \mathrm{m}^{2}\right)^{\mathrm{a}}(n=4092)$ & 19.1 & 18.9 & 19.3 & 19.0 & 18.8 & 19.3 & 18.9 & 18.6 & 19.1 & 19.0 & 18.7 & 19.2 & $32.93^{*}$ & 0.45 & 0.001 \\
\hline$W C(\mathrm{~cm})^{\mathrm{a}}(n=4076)$ & 67.8 & 67.2 & 68.4 & 67.4 & 66.7 & 68.0 & $66.6 \neq$ & 66.0 & 67.1 & 66.8 & 66.1 & 67.6 & $61.71^{*}$ & 0.03 & 0.002 \\
\hline $\begin{array}{l}\text { Physical Competence } \\
\text { domain score }^{b}(n=4092)\end{array}$ & 19.9 & 19.7 & 20.2 & $19.5 \neq$ & 19.3 & 19.7 & $19.2 \ddagger$ & 19.0 & 19.3 & $18.9 \neq \dagger$ & 18.7 & 19.2 & $276.74^{*}$ & $<0.001$ & 0.01 \\
\hline $\begin{array}{l}\text { Daily Behaviour domain } \\
\text { score }^{b}(n=4092)\end{array}$ & 18.5 & 18.0 & 18.9 & 18.2 & 17.8 & 18.7 & 18.0 & 17.6 & 18.4 & 18.5 & 18.1 & 19.0 & $16.08^{*}$ & 0.34 & 0.001 \\
\hline $\begin{array}{l}\text { Motivation and Confidence } \\
\text { domain score }^{b}(n=4092)\end{array}$ & 12.5 & 12.3 & 12.6 & 12.3 & 12.1 & 12.4 & $12.2 \ddagger$ & 12.0 & 12.3 & $12.2 \neq$ & 12.0 & 12.3 & $8.72^{*}$ & 0.036 & 0.002 \\
\hline $\begin{array}{l}\text { Knowledge and Understanding } \\
\text { domain score }{ }^{\mathrm{a}}(n=4133)\end{array}$ & 12.4 & 12.2 & 12.6 & 12.2 & 12.0 & 12.3 & 12.2 & 12.1 & 12.4 & $11.9 \neq$ & 11.8 & 12.1 & $72.83^{*}$ & 0.001 & 0.004 \\
\hline Total CAPL score ${ }^{\mathrm{b}}(n=4092)$ & 63.3 & 62.6 & 63.9 & 62.1 & 61.5 & 62.1 & $61.5 \ddagger$ & 60.9 & 62.1 & $61.6 \neq$ & 60.9 & 62.3 & $49.56^{*}$ & 0.001 & 0.004 \\
\hline
\end{tabular}

An analysis of covariance was conducted controlling for multiple covariates described in each model. The $F$-test is shown for the overall corrected model

$\left({ }^{*} p<0.001\right)$. The last two columns, the $p$-value and Cohen's $f^{2}$ effect sizes, are presented for the main effect of the relative age by quarters

${ }^{\ddagger}$ statistically significant differences between quarter 1 and other quarters

${ }^{\dagger}$ statistically significant differences between quarter 2 and other quarters

acontrolled for site location, age (whole years) and testing month

${ }^{\mathrm{b}}$ controlled for BMI $z$-score, site location, age (whole years) and testing month

CI confidence interval, $L B$ lower bound, UB upper bound, BMI body mass index, WC waist circumference, CAPL Canadian Assessment of Physical Literacy

No RAE was observed for the Daily Behaviour domain scores $(F(3,4051)=1.4, p=0.26$ and $F(3,4084)=1.1, p$ $=0.34$ in boys and girls, respectively). Interestingly, the largest contributor in the model in boys for this domain score was the covariate BMI $z$-score $(F(1,4051)=51.4$, $p<0.001)$ while in girls the largest contributor was covariate age $(F(1,4084)=1.1, p=0.34)$. In terms of effect sizes, the BMI $z$-score covariate in boys was negligible $\left(f^{2}=0.01\right)$ but in the girls' model the covariate age produced a small effect size $\left(f^{2}=0.02\right)$.

Girls' Motivation and Confidence domain scores were significantly associated with the relative age $(F(3,4084)$ $=2.9, p=0.04)$, but the association's effect size was negligible. Among boys and girls, the Knowledge and Understanding domain scores were also significantly associated with relative age $(F(3,4093)=9.8, p<0.0001$ and $F(3,4126)=5.2, p=0.001$, respectively). Again, these main effect associations produced negligible effect sizes, while the covariate age in the association between relative age and the Knowledge and Understanding domain scores generated small effect sizes $\left(f^{2}=0.10\right.$ and $f^{2}=$ 0.09 in boys and girls, respectively).

Among both genders, there was a significant main effect of birth month, in quarters, on the overall CAPL scores $(F(3,4051)=2.7, p=0.04$ and $F(3,4084)=5.8, p$ $=0.001$ in boys and girls, respectively). The effect sizes 


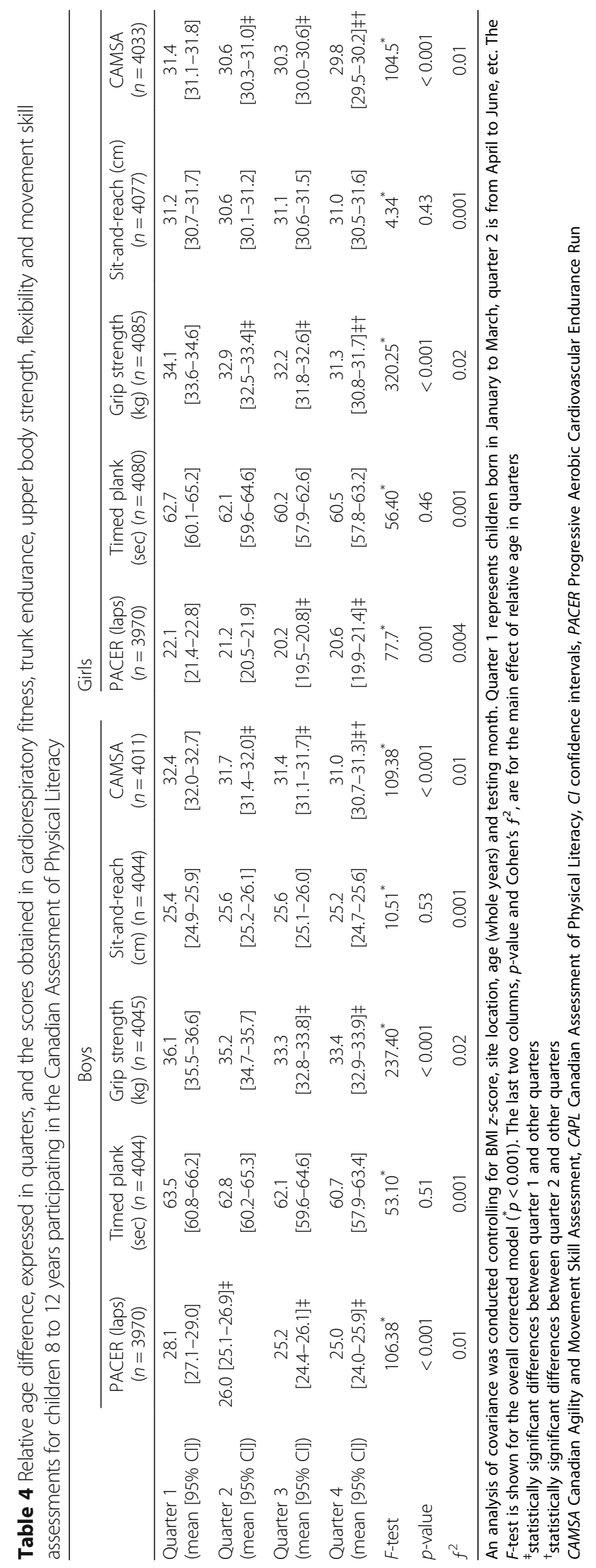


for the associations between the overall CAPL scores and the relative age were negligible. The covariate BMI $z$-score was the largest contributor in the model, even larger than the age covariate, for the overall CAPL scores in both boys and girls $(F(1,4051)=383.1, p<$ $0.0001, f^{2}=0.09$ and $F(1,4084)=324.7, p<0.0001, f^{2}=$ 0.08 , respectively). The associations between children's relative age, in quarters, and the specific Physical Competence assessment protocol are presented in Table 4 . Despite having a statistically significant corrected model for all the assessments, the main effect of quarters was only statistically significant for children's cardiorespiratory fitness (PACER shuttle run test), the upper body strength assessment (grip strength), and the movement skill assessment (CAMSA). Among boys and girls, the number of 20-m laps run was associated with the relative age of children $(F(3,3962)=8.6, p<0.0001$ and $F(3$, $3962)=5.8, p=0.001$, respectively). However, this significant main effect of relative age for the cardiorespiratory fitness assessment revealed only negligible effect sizes. While the main effect for the cardiorespiratory fitness assessment was negligible, the covariate BMI $z$-score contributed to moderate $\left(f^{2}=0.16\right)$ and small $\left(f^{2}=0.11\right)$ effect sizes in the model in boys and girls, respectively. The significant associations between the movement skills assessment (CAMSA) and the relative age among boys and girls $(F(3,4003)=11.0, p<0.001$ and $F(3,4025)=$ $15.8, p<0.001$, respectively) also revealed only negligible effect sizes. While effect sizes were negligible for both the cardiorespiratory fitness and the movement skills assessment, the significant association between upper body strength (handgrip strength) and relative age $(F(3,4037)$ $=29.2, p<0.001$ and $F(3,4077)=25.1, p<0.001$ in boys and girls, respectively) revealed small effect sizes in both genders. Among boys and girls, neither the muscular endurance (timed plank) $(F(3,4063)=0.8, p=0.51$ and $F(3,4072)=0.87, p=0.46$, respectively) nor the flexibility (sit-and-reach) $(F(3,4036)=0.7, p=0.53$ and $F(3,4069)$ $=0.4, p=0.43$, respectively) assessments were associated with the relative age in quarters.

\section{Discussion}

The aim of our study was to quantify the magnitude of the RAE as it relates to CAPL scores (physical literacy) in a large sample of children aged 8 to 12 years. Although we found many significant associations between the domain scores and relative age expressed in quarters, the RAE bias was negligible on the domains and overall CAPL scores. Additionally, we also observed a small relative age association among boys' and girls' height and strength measurements. Boys born in the first three months of the year were taller and heavier, and had higher handgrip strength, compared to those born later in the same year. Girls born in the first six months after the school entry cut-off date were taller and had higher handgrip strength compared to their relatively younger peers born in the last six months of the year.

\section{Anthropometrics}

Several studies in sports have hypothesised that the RAE could be attributed to physical advantages based on growth differences [9, 10, 43]. However, this hypothesis is not supported by all the research. In the sport context, a number of studies have observed that relatively older children were significantly taller than those born later in the same year [43-48]; however, others reported no significant associations between relative age difference and anthropometric measurements $[49,50]$. Studies examining the association between children's RAE and fitness or fundamental movement skills assessments outside of a specific sport context have also reported inconsistent RAE results for anthropometric measurements. For instance, Sandercock and colleagues [23] found no association between the RAE and the anthropometrics of their participants. In the present study, we did observe a RAE in children's height mainly; children born in the first six months of the year were taller than their peers born in the last six months of that same year. The present study's results are consistent with a previous study [11]. However, these anthropometric advantages seen in the relatively older children did not appear to have much influence on their physical literacy scores.

\section{Domain scores and overall CAPL scores}

Gender differences were observed in the RAE, particularly evident in the Motivation and Confidence domain scores. Boys' scores were not significantly different based on the main effect of relative age, while girls born in the first three months of the year obtained greater scores in the Motivation and Confidence domain than those born in the last six months of the same year. Previous studies have shown that boys generally display greater self-efficacy and motivation, but also receive greater social support toward physical activities and sports than girls [51, 52]. These gender differences in the psychosocial correlates of physical activity may be partly responsible for the lack of association between the RAE in boys' Motivation and Confidence scores. Another plausible explanation may involve biological maturation and its association to physical self-concepts [53, 54]. Physical self-concept is considered to be both a determinant and outcome of physical activity, with an increased positive self-concept being positively associated with daily physical activity [55]. In the present study, the adequacy component within the Motivation and Confidence domain was the only component that was significantly associated with the relative age in girls $\left(F(3,4084)=5.5, p=0.01, f^{2}=\right.$ $0.004)$. Adequacy refers to a generalized self-efficacy toward physical activity [38], and being consistently older in school and in sports cohorts may have contributed to the increased level of self-efficacy in the relatively older girls. Both 
biological maturity status and self-concepts, unmeasured in the present study, could have influenced children's scores on the Motivation assessment.

Evidence of the RAE in the affective domain is very limited. Thompson and colleagues [16] investigated the association between the RAE of Grade 1 children and self-esteem, and found a positive association between relatively older children and greater self-esteem in school. Although the study was conducted in a classroom setting and in younger children, no gender difference in the relationship between self-esteem and RAE was reported [16]. More research should investigate the association between gender, biological maturity, relative age and different affective outcomes (i.e., motivation, self-efficacy, self-concept, and confidence), since these factors may impact long-term physical activity participation.

Numerous studies have reported on the relationship between a child's month of birth and academic abilities. Relatively older children tend to consistently score higher on school tests than their relatively younger peers throughout their education $[13,56-60]$. In the present study, the RAE results on the association with the Knowledge and Understanding domain scores are consistent with previous studies despite the negligible effect sizes observed. It is important to note that boys born in the first six months of the year scored higher in the Knowledge and Understanding domain than their peers born in the last six months of the year, while girls born in the first three months of the year outscored their peers born in the last three months of the year. In contrast with a recent study [56], the present study's RAE association with the Knowledge and Understanding domain scores was not attenuated in older children (11 and 12 years old), even though all children completed the same questionnaire. These findings may further substantiate Boardman's theory [59] that relatively younger children may have different and unmet learning needs than their relatively older peers.

In the present study, while just short of a small effect size, a relative age difference was observed in the Physical Competence domain scores among both genders. Roberts and colleagues [11] have hypothesized that the lower physical fitness they observed among relatively younger children may be due to less daily physical activity. If this were the case, we would have observed a RAE in the Daily Behaviour domain components (i.e., daily step counts and the self-reported number of days a child engages in moderate to vigorous physical activity per week); however, we observed no significant differences between birth months and the individual domain components or the Daily Behaviour domain scores. In fact, the highest Daily Behaviour domain score was seen in boys born in the last three months of the year. Therefore, the amount of daily physical activity does not appear to be a factor in the RAE bias; a more plausible explanation would be the maturation differences and the positive linear relationship between age and performance in this age group [11, 61].

Relative age differences were statistically significant in both boys (two of four domains) and girls (three of four domains); however, these associations yielded negligible effect sizes. Thus, regarding the overall CAPL score, the observed negligible effect sizes were not surprising considering the aggregate nature of the CAPL scoring system. For the domains and the overall CAPL scores, the covariate BMI $z$-score had a greater impact than the relative age, in quarters, for the Physical Competence domain (large effect sizes) and overall CAPL scores (small effect sizes) in both genders. The greater impact observed for the BMI $z$-score covariate could be partially explained by our participants' mean age and the BMI $z$-score acting as a proxy measure of maturation, as the World Health Organization's BMI $z$-score are age- (in years and months) and gender- specific [28]. Additionally, the covariate age had a greater impact than the relative age on the Knowledge and Understanding domain (small effect sizes) in both genders, and on the Daily Behaviour domain in girls (small effect sizes).

\section{Physical competence fitness assessments}

Among boys and girls, the strength assessment showed evidence of a RAE bias but the effect sizes were deemed small. The RAE associated with the handgrip strength test is also consistent with a previous study [23]. In this age group, rounded-down age would seem to unfairly compare strength in both genders; this may be related to height and muscle mass differences. It may be worth investigating whether the handgrip strength test is associated with physical literacy or with fitness in this age group, since our results suggest that handgrip strength is highly susceptible to both the children's anthropometrics (possibly a result of biological maturation variation) and the RAE bias. A nationally representative study conducted in the United States on the association between body weight and strength measurements among children 6 to 15 years of age has observed a positive association between grip strength and weight status, where children who were considered overweight and obese outperformed children of ideal weight [62]. The latter study also reinforced the argument that handgrip strength may be a poor assessment of musculoskeletal fitness in children. Moreover, the poor association between handgrip strength and children' physical literacy is further discussed by Gunnell and colleagues [63].

Boys born in the first quarter ran significantly more laps compared to their relatively younger peers, while in girls only the comparison between those born in the first quarter and the last six months was significant. While the effect sizes were negligible for the PACER shuttle run test, the more pronounced relative age difference in boys' cardiorespiratory fitness may be a product of the sample mean age; at 10 years 
the majority of boys would be prepubescent, while there is an increased likelihood of having more variation in the biological maturation stage of girls [64-66]. The observation of a relative age difference in cardiorespiratory fitness is consistent with previous studies [11, 23, 61].

Among both boys and girls, the CAMSA, a measure of children's movement skills, was significantly associated with the relative age difference of children. This study's results showed significant associations between the relative age and the movement skill assessment in both genders, but the main effect of relative age showed negligible effect sizes. These significant associations between RAE and movement skill assessment are consistent with a recent study by Birch and colleagues [22] on the association between the RAE and fundamental movement skills assessments (effect sizes not reported). In contrast with that study, however, we did not observe gender differences in the CAMSA mean scores. The CAMSA produces an overall skill score that could be covering for gender differences in object control versus balance tasks, for example. Birch and colleagues' observation of gender differences in object control in favour of boys is consistent with findings from a recent systematic review and meta-analysis of the correlates of gross motor skills in children between 3 and 18 years of age [67].

Even though the results for the trunk muscular endurance measurement (timed plank) were not significantly associated with the RAE, the scores did follow the expected pattern for the RAE (quarter $1>$ quarter $2>$ quarter $3>$ quarter 4). However, the expected RAE pattern was not observed for the flexibility assessment (sit-and-reach). The scores were significantly different between boys and girls but no significant differences were observed between whole age groups in girls, whereas younger boys (i.e., ages 8 and 9) scored significantly higher than older boys (data not shown). These observed age and gender differences and scoring patterns in the sit-and-reach are consistent with previous studies observing children's fitness $[68,69]$.

Strengths of the present study include the large sample size and the harmonized, validated, population-specific, and age- and gender-normalized protocol that measured physical literacy of children across 11 sites across Canada. Another strength of our study was the high inclusion rate (between 79 and $82 \%$ for all samples), despite the need for complete assessment, domain scores and overall CAPL scores. Finally, we believe that this study presents a realistic observation of the RAE bias, in a physical literacy context, as not all studies exploring this bias have reported effect sizes to determine if any differences observed are in fact meaningful.

However, these results need to be interpreted in light of the following limitations. First, because our data are cross-sectional, causality cannot be established. Second, the external generalizability of these findings may be limited due to the nonprobability sampling strategy, which may have produced inflated physical literacy scores. However, our sample birth distribution (in quarters) was representative of the Canadian population birth distribution [42]. Finally, having some information on biological maturation status (e.g., measuring sitting height) would have provided some additional insight that might have helped to confirm some of the theories we advanced to explain our results.

\section{Conclusions}

Collectively, the associations between children's relative age and the CAPL domain and overall scores produced mainly negligible effect sizes, suggesting that the RAE is not an important factor to consider when assessing the physical literacy of children with the CAPL. In practice, the mean differences observed across birth months, in quarters, were not large enough to warrant an adjustment to the CAPL scores. However, we believe that it is good practice to explore possible RAE bias in new assessment protocols, specifically those that separate children into rounded-down age bands, which may or may not be appropriate for all age groups. As these age bands do not take into account length of time in school, relatively older children are likely to have greater skill development opportunities based on school entry cut-off dates than their peers born later in the year. The lack of RAE findings is positive for the CAPL assessment, since the CAPL's development was partially informed by schools' curricula; thus the potential for a RAE warranted exploration. This study is unique as it provides a comprehensive examination of the RAE that assessed the association in affective, cognitive, behavioural, and physical domains of physical literacy in children.

\section{Additional files}

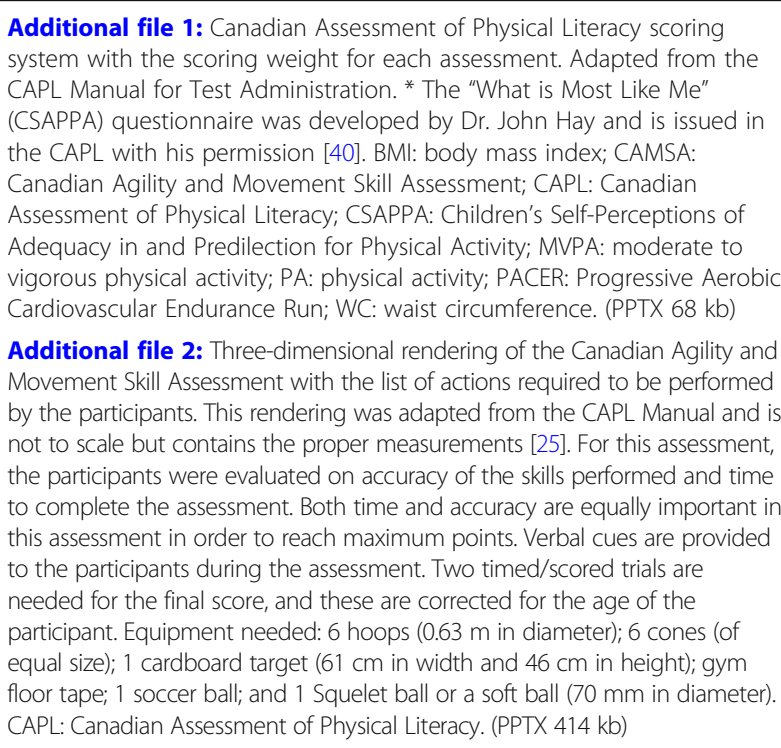

Additional file 2: Three-dimensional rendering of the Canadian Agility and Movement Skill Assessment with the list of actions required to be performed by the participants. This rendering was adapted from the CAPL Manual and is not to scale but contains the proper measurements [25]. For this assessment, the participants were evaluated on accuracy of the skills performed and time to complete the assessment. Both time and accuracy are equally important in this assessment in order to reach maximum points. Verbal cues are provided to the participants during the assessment. Two timed/scored trials are needed for the final score, and these are corrected for the age of the participant. Equipment needed: 6 hoops ( $0.63 \mathrm{~m}$ in diameter); 6 cones (of equal size); 1 cardboard target ( $61 \mathrm{~cm}$ in width and $46 \mathrm{~cm}$ in height); gym floor tape; 1 soccer ball; and 1 Squelet ball or a soft ball (70 $\mathrm{mm}$ in diameter). CAPL: Canadian Assessment of Physical Literacy. (PPTX 414 kb) 


\section{Abbreviations}

ANCOVA: analysis of covariance; BMl: body mass index; CAMSA: Canadian Agility and Movement Skill Assessment; CAPL: Canadian Assessment of Physical Literacy; CSAPPA: Children's Self-Perceptions of Adequacy in and Predilection for Physical Activity; PACER: Progressive Aerobic Cardiovascular Endurance Run; RAE: relative age effect; RBC: Royal Bank of Canada; WC: waist circumference

\section{Acknowledgements}

The authors would like to acknowledge and thank the principal investigators of all the RBC-CAPL Learn to Play project sites: Kristal D. Anderson, Brenda Bruner, Jennifer L. Copeland, Claude Dugas, Melanie J. Gregg, Nathan Hall, Angela M. Kolen, Kirstin N. Lane, Barbi Law, Dany J. MacDonald, Luc J. Martin, Travis J. Saunders, Dwayne Sheehan, Michelle Stone, François Trudeau and Sarah J. Woodruff. Without them this research would not have been possible.

\section{Funding}

This study was funded by the Royal Bank of Canada, the Public Health Agency of Canada, and Mitacs, and was delivered in partnership with ParticipACTION. Publication charges for this article have been funded by the RBC Learn to Play project and the Public Health Agency of Canada, delivered in partnership with ParticipACTION.

\section{Availability of data and materials}

The datasets supporting the conclusions of this article are available upon reasonable request to Dr. Mark S. Tremblay (mtremblay@cheo.on.ca).

\section{About this supplement}

This article has been published as part of BMC Public Health Volume 18 Supplement 2, 2018: Canadian Assessment of Physical Literacy. The full contents of the supplement are available online at https//bmcpublichealth.biomedcentral.com/ articles/supplements/volume-18-supplement-2.

\section{Authors' contributions}

CD, MST and J-PC developed the research question and objectives. CD, JDB and J-PC led the data analysis and synthesis of results. CD wrote the manuscript. CD, MST, PEL, JDB, KB and J-PC participated in data collection and were responsible for reviewing, and revising the manuscript for important intellectual content. All authors read and approved the final manuscript.

\section{Ethics approval and consent to participate}

Ethics approval was obtained from: Antigonish - St. Francis University Research Ethics Board and the Strait Regional School Board; Calgary - Mount Royal University Human Research Ethics Board; Charlottetown - University of Prince Edward Island Research Ethics Board and the Prince Edward Island Public Schools Branch Research Ethics Board; Halifax - Dalhousie University Research and Ethics Board and the Halifax Regional School Board; Lethbridge - University of Lethbridge Human Subject Research Committee; North Bay - Nipissing University Research Ethics Board, Near North District School Board, Nipissing Parry Sound Catholic District School Board, and Conseil Scolaire Catholique Franco-Nord; Ottawa - Children's Hospital of Eastern Ontario Research Ethics Board, University of Ottawa Research Ethics Board, Ottawa-Carleton District School Board, Ottawa Catholic School Board, Conseil des écoles catholiques du Centre-Est, Conseil des écoles publiques de l'Est de l'Ontario, Upper Canada District School Board, Durham District School Board, University of Illinois at Urbana-Champaign; Trois-Rivières - Université du Québec à Trois-Rivières Research Ethics Board; Victoria - Camosun College Research Ethics Board and the Greater Victoria School District; Windsor - University of Windsor Research Ethics Board and the Windsor Essex Catholic District School Board; Winnipeg The University of Winnipeg University Human Research Ethics Board (UHREB), River East Transcona School Division, and St. James-Assiniboia School Division. Written informed consent was obtained from parents or legal guardians, and participating children also provided verbal assent.

\section{Consent for publication}

Not applicable.

\section{Competing interests}

The authors declared that they have no conflicts of interest.

\section{Publisher's Note}

Springer Nature remains neutral with regard to jurisdictional claims in published maps and institutional affiliations.

Published: 2 October 2018

\section{References}

1. International Physical Literacy Association homepage. 2016. https://www. physical-literacy.org.ukJ. Accessed 3 Apr 2017.

2. Poitras VJ, Gray CE, Borghese MM, Carson V, Chaput J-P, Janssen I, et al. Systematic review of the relationships between objectively measured physical activity and health indicators in school-aged children and youth. Appl Physiol Nutr Metab. 2016:41:S197-239.

3. Castelli DM, Barcelona JM, Bryant L. Contextualizing physical literacy in the school environment: the challenges. J Sport Heal Sci. 2015;4:156-63.

4. Giblin S, Collins D, Button C. Physical literacy: importance, assessment and future directions. Sport Med. 2014;44:1177-84.

5. ParticipACTION, Canadian Sport for Life Society, the Healthy Active Living and Obesity Research Group at the Children's Hospital of Eastern Ontario, Physical Health and Education Canada, Canadian Parks and Recreation Association, Ontario Society of Physical Activity Promoters in Public Health. Canada's Physical Literacy Consensus Statement, June 2015. 2015. https:// www.participaction.com/sites/default/files/downloads/ParticipactionCanadianPhysicalLiteracy-Consensus_0.pdf. Accessed 3 Apr 2017.

6. Francis $C E$, Longmuir PE, Boyer C, Andersen LB, Barnes JD, Boiarskaia E, et al. The Canadian Assessment of Physical Literacy: development of a model of children's capacity for a healthy, active lifestyle through a Delphi process. J Phys Act Heal. 2016;13:214-22.

7. Longmuir PE, Boyer C, Lloyd M, Yang Y, Boiarskaia E, Zhu W, Tremblay MS. The Canadian Assessment of Physical Literacy: methods for children in grades 4 to 6 (8 to 12 years). BMC Public Health. 2015;15:767-88.

8. Bell JF, Daniels S. Are summer-born children disadvantaged? The birthdate effect in education. Oxford Rev. Educ. 1990;16:67-80

9. Cobley S, Baker J, Wattie N, McKenna J. Annual age-grouping and athlete development: a meta-analytical review of relative age effects in sports. Sports Med. 2009;39:235-56.

10. Musch J, Grondin S. Unequal competition as an impediment to personal development: a review of the relative age effect in sport. Dev Rev. 2001;21: $147-67$.

11. Roberts SJ, Boddy LM, Fairclough SJ, Stratton G. The influence of relative age effects on the cardiorespiratory fitness levels of children age 9 to 10 and 11 to 12 years of age. Pediatr Exerc Sci. 2012;24:72-83.

12. Bisanz J, Morrison FJ, Dunn M. Effects of age and schooling on the acquisition of elementary quantitative skills. Dev Psychol. 1995;31:221-36.

13. Campbell T. Stratified at seven: in-class ability grouping and the relative age effect. Br Educ Res J. 2014;40:749-71.

14. Roberts SJ, Fairclough SJ. The influence of relative age effect in the assessment of high school students in physical education in the United Kingdom. J Teach Phys Educ. 2012;31:56-70

15. Sykes EDA, Bell JF, Vidal RC. Birthdate effects: a review of the literature from 1990-on. Cambridge: Cambridge Assessment, University of Cambridge Local Examination Syndicate; 2016.

16. Thompson AH, Barnsley RH, Battle J. The relative age effect and the development of self-esteem. Educ Res. 2004:46:313-20.

17. Patalay P, Belsky J, Fonagy P, Vostanis $\mathrm{P}$, Humphrey N, Deighton J, Wolpert $M$. The extent and specificity of relative age effects on mental health and functioning in early adolescence. J Adolesc Health. 2015;57:475-81.

18. Dhuey E, Lipscomb S. Disabled or young? Relative age and special education diagnoses in schools. Econ Educ Rev. 2010;29:857-72.

19. Crane J, Temple V. A systematic review of dropout from organized sport among children and youth. Eur Phys Educ Rev. 2015;21:114-31.

20. Delorme N, Chalabaev A, Raspaud M. Relative age is associated with sport dropout: Evidence from youth categories of French basketball. Scand J Med Sci Sports. 2011;21:120-8.

21. Morrow RL, Garland EJ, Wright JM, Maclure M, Taylor S, Dormuth CR. Influence of relative age on diagnosis and treatment of attention-deficit/ hyperactivity disorder in children. Can Med Assoc J. 2012;184:755-62.

22. Birch $S$, Cummings $L$, Oxford SW, Duncan MJ. Examining relative age effects in fundamental skill proficiency in British children aged 611 years. J Strength Cond Res. 2016;30:2809-15. 
23. Sandercock GR, Taylor MJ, Voss C, Ogunleye AA, Cohen DD, Parry DA. Quantification of the relative age effect in three indices of physical performance. J Strength Cond Res. 2013;27:3293-9.

24. Veldhuizen S, Rivard L, Cairney J. Relative age effects in the Movement Assessment Battery for Children-2: age banding and scoring errors. Child Care Health Dev. 2017:1-6. [Epub 2017 Mar 15].

25. Healthy Active Living and Obesity Research Group. Canadian Assessment of Physical Literacy: manual for test administration. Ottawa: Healthy Active Living and Obesity Research Group; 2013 https://www.capl-ecsfp.ca/wp-content/uploads/capl-manual-english.pdf. Accessed 3 Apr 2017

26. Tremblay MS, Costas-Bradstreet C, Barnes JD, Bartlett B, Dampier D, Lalonde C, et al. Canada's Physical Literacy Consensus Statement: process and outcome. BMC Public Health. 2018;18(Suppl 2). https:/doi.org/10.1186/s12889-018-5903-X.

27. Tremblay MS, Longmuir PE, Barnes JD, Belanger K, Anderson KD, Bruner $\mathrm{B}$, et al. Physical literacy levels of Canadian children aged 8-12 years: descriptive and normative results from the RBC Learn to Play-CAPL project. BMC Public Health. 2018;18(Suppl 2). https://doi.org/10.1186/ s12889-018-5891-x.

28. de Onis M, Onyango AW, Borghi E, Siyam A, Siekmann J. Development of a WHO growth reference for school-aged children and adolescents. Bull World Health Organ. 2007;85:660-7

29. CSEP. Canadian Society for Exercise Physiology - Physical Activity Training for Health. CSEP: Ottawa; 2013.

30. Carrel AL, Bowser J, White D, Moberg DP, Weaver B, Hisgen J, et al. Standardized childhood fitness percentiles derived from school-based testing. J Pediatr. 2012;161:120-4.

31. Boyer C, Tremblay MS, Saunders TJ, McFarlane A, Borghese M, Lloyd M, Longmuir PE. Feasibility, validity and reliability of the plank isometric hold as a field-based assessment of torso muscular endurance for children 812 years of age. Pediatr Exerc Sci. 2013;25:407-22.

32. Longmuir PE, Boyer C, Lloyd M, Borghese MM, Knight E, Saunders TJ, et al. Canadian Agility and Movement Skill Assessment (CAMSA): validity, objectivity, and reliability evidence for children 8-12 years of age. J Sport and Health Sci. 2015:231-40.

33. Rowe DA, Mahar MT, Raedeke TD, Lore J. Measuring physical activity in children with pedometers: reliability, reactivity, and replacement of missing data. Pediatr Exerc Sci. 2004;16:1-12.

34. Eisenmann JC, Laurson KR, Wickel EE, Gentile D, Walsh D. Utility of pedometer step recommendations for predicting overweight in children. Int J Obes (Lond). 2007;31:1179-82.

35. Tudor-Locke C, Burkett L, Reis JP, Ainsworth BE, Macera CA, Wilson DK. How many days of pedometer monitoring predict weekly physical activity in adults? Prev Med. 2005;40:293-8.

36. Saunders TJ, MacDonald DJ, Copeland JL, Longmuir PE, Barnes JD, Belanger $K$, et al. The relationship between sedentary behaviour and physical literacy in Canadian children: a cross-sectional analysis from the RBC-CAPL Learn to Play study. BMC Public Health. 2018;18(Suppl 2). https://doi.org/10.1186/ s12889-018-5892-9.

37. Longmuir PE, Woodruff SJ, Boyer C, Lloyd M, Tremblay MS. Physical Literacy Knowledge Questionnaire: feasibility, validity, and reliability for Canadian children aged 8 to 12 years. BMC Public Health. 2018;18(Suppl 2). https:// doi.org/10.1186/s12889-018-5890-y.

38. MacDonald DJ, Saunders TJ, Longmuir PE, Barnes JD, Belanger K, Bruner B, et al. A cross-sectional study exploring the relationship between age, gender, and physical measures with adequacy in and predilection for physical activity. BMC Public Health. 2018;18(Suppl 2). https:/doi.org/10.1186/s12889-018-5893-8.

39. Garcia AW, Broda MA, Frenn M, Coviak C, Pender NJ, Ronis DL. Gender and developmental differences in exercise beliefs among youth and prediction of their exercise behavior. J Sch Health. 1995;65:213-9.

40. Hay JA. Adequacy in and predilection for physical activity in children. Clin J Sport Med. 1992;2:192-201.

41. Cohen J. Statistical power analysis for the behavioral sciences. 2nd ed. Statistical Power Analysis for the Behavioral Sciences. Hilldale: Lawrence Erlbaum Associates; 1988.

42. Statistics Canada. CANSIM (database). Table 102-4502 - Live births, by month, Canada, provinces and territories. 2017. http://www5.statcan.gc.ca/ cansim/a26?lang=eng\&id=1024502. Accessed 19 Apr 2017.

43. Carling C, Le Gall F, Reilly T, Williams AM. Do anthropometric and fitness characteristics vary according to birth date distribution in elite youth academy soccer players? Scand J Med Sci Sport. 2009;19:3-9.
44. Roczniok R, Maszczyk A, Stanula A, Czuba M, Pietraszewski P, Kantyka J, Starzynski MA. Physiological and physical profiles and on-ice performance approach to predict talent in male youth ice hockey players during draft to hockey team. Isokinet Exerc Sci. 2013;21:121-7.

45. Gil SM, Badiola A, Bidaurrazaga-Letona I, Zabala-Lili J, Gravina L, SantosConcejero J, et al. Relationship between the relative age effect and anthropometry, maturity and performance in young soccer players. J Sports Sci. 2014;32:479-86.

46. Hirose N. Relationships among birth-month distribution, skeletal age and anthropometric characteristics in adolescent elite soccer players. J Sports Sci. 2009;27:1159-66.

47. Torres-Unda J, Zarrazquin I, Gil J, Ruiz F, Irazusta A, Kortajarena M, et al. Anthropometric, physiological and maturational characteristics in selected elite and non-elite male adolescent basketball players. J Sports Sci. 2013;31:196-203.

48. Müller $L$, Müller $E$, Rashner $C$. The relative age effect in alpine ski racing: a review. Talent Dev Excell. 2016;8:3-14.

49. Malina RM, Ribeiro B, Aroso J, Cumming SP, Unnithan V, Kirkendall D. Characteristics of youth soccer players aged 13-15 years classified by skill level. Br J Sports Med. 2007;41:290-5.

50. Deprez D, Vaeyens R, Coutts AJ, Lenoir M, Philippaerts R. Relative age effect and Yo-Yo IR1 in youth soccer. Int J Sports Med. 2012;33:987-93.

51. Van Der Horst K, Paw MJCA, Twisk JWR, Van Mechelen W. A brief review on correlates of physical activity and sedentariness in youth. Med Sci Sports Exerc. 2007;39:1241-50.

52. Cardon G, Philippaerts R, Lefevre J, Matton L, Wijndaele K, Balduck A-L, De Bourdeaudhuij I. Physical activity levels in 10- to 11-year-olds: clustering of psychosocial correlates. Public Health Nutr. 2005;8:896-903.

53. Baxter-Jones ADG, Eisenmann JC, Sherar LB. Controlling for maturation in pediatric exercise science. Pediatr Exerc Sci. 2005;17:18-30.

54. Cumming SP, Standage M, Loney T, Gammon C, Neville H, Sherar LB, Malina $\mathrm{RM}$. The mediating role of physical self-concept on relations between biological maturity status and physical activity in adolescent females. J Adolesc. 2011:34:465-73.

55. Sabiston CM, Crocker PRE. Exploring self-perceptions and social influences as correlates of adolescent leisure-time physical activity. J Sport Exerc Psychol. 2008:30:3-22.

56. Navarro J-J, García-Rubio J, Olivares PR. The relative age effect and its influence on academic performance. PLoS One. 2015;10:1-18.

57. Lawlor DA, Clark H, Ronalds G, Leon DA. Season of birth and childhood intelligence: findings from the Aberdeen Children of the 1950s-cohort study. Br J Educ Psychol. 2006;76:481-99.

58. Bedard K, Dhuey E. The persistence of early childhood maturity: international evidence of long-run age effects. Q J Econ. 2006;121:1437-72.

59. Boardman M. The impact of age and gender on prep children's academic achievements. Aust J Early Child. 2006;31:1-6.

60. Menet F, Eakin J, Stuart M, Rafferty H. Month of birth and effect on literacy, behaviour and referral to psychological service. Educ Psychol Pract. 2000;16:225-34.

61. Veldhuizen S, Cairney J, Hay J, Faught B. Relative age effects in fitness testing in a general school sample: how relative are they? J Sports Sci. 2015; 33:109-15.

62. Ervin BR, Fryar CD, Wang C-Y, Miller IM, Ogden CL. Strength and body weight in US children and adolescents. Pediatr. 2014;134:e782-9.

63. Gunnell KE, Longmuir PE, Barnes JD, Belanger K, Tremblay MS. Refining the Canadian Assessment of Physical Literacy based on theory and factor analyses. BMC Public Health. 2018;18(Suppl 2). https://doi.org/10.1186/ s12889-018-5899-2.

64. Abbassi V. Growth and normal puberty. Pediatr. 1998;102:507-11.

65. Thissen D, Bock RD, Wainer H, Roche AF. Individual growth in stature: a comparison of four growth studies in the U.S.A. Ann Hum Biol. 1976;3:529-42.

66. Jones MA, Hitchen PJ, Stratton G. The importance of considering biological maturity when assessing physical fitness measures in girls and boys aged 10 to 16 years. Ann Hum Biol. 2000;27:57-65.

67. Barnett LM, Lai SK, Veldman SLC, Hardy LL, Cliff DP, Morgan PJ, et al. Correlates of gross motor competence in children and adolescents: a systematic review and meta-analysis. Sports Med. 2016;46:1663-88.

68. Tremblay MS, Shields M, Laviolette M, Craig CL, Janssen I, Connor Gorber S. Fitness of Canadian children and youth: results from the 2007-2009 Canadian Health Measures Survey. Health Rep. 2010;21:7-20.

69. Marta CC, Marinho DA, Barbosa TM, Izquierdo M, Marques MC. Physical fitness differences between prepubescent boys and girls. J Strength Cond Res. 2012;26:1756-66. 